\title{
The importance of treating mild hyperglycemia in pregnant women with diabetes
}

\author{
Kyung-Soo Kim
}

Department of Internal Medicine, CHA Bundang Medical Center, CHA University, Seongnam, Korea

Received: September 28, 2018 Accepted: October 12, 2018

\author{
Correspondence to \\ Kyung-Soo Kim, M.D. \\ Department of Internal Med- \\ icine, CHA Bundang Medical \\ Center, CHA University, 59 Yat- \\ ap-ro, Bundang-gu, Seongnam \\ 13496, Korea \\ Tel: +82-31-780-4849 \\ Fax: +82-31-780-5208 \\ E-mail: kks982@hanmail.net
}

\section{See Article on Page 1143-1149}

The risk for hyperglycemia in pregnancy is increasing, because the prevalence of obesity has been increasing in women of childbearing age $[1,2]$. Maternal hyperglycemia, including gestational diabetes mellitus (GDM) and pregestational diabetes, occurs in $3 \%$ to $10 \%$ of pregnancies, with GDM accounting for the majority of cases (approximately 90\%) and preexisting diabetes making up the remainder $[2,3]$. In general, women with GDM have abnormal maternal outcomes (i.e., preeclampsia, higher rates of cesarean section, and high rates of macrosomia) and neonatal outcomes (i.e., respiratory distress syndrome, neonatal hypoglycemia, and hyperbilirubinemia) [4-6]. Furthermore, pregestational diabetes in pregnancy confers significantly greater risks, such as fetal anomalies and fetal demise, than GDM $[7,8]$. Consequently, tight glycemic control is critical to reduce perinatal and neonatal risks in pregnant women with diabetes.

As reported in the current issue, Jang et al. [9] compared maternal and neonatal outcomes in Korean women with type 2 diabetes and nondiabetic controls. Although the rate of major congenital malformations and the risk of being small for gestational age did not differ, women with type 2 diabetes showed a slightly higher risk for primary cesarean section, preeclampsia, infections during pregnancy, high neonatal weight, being large for gestational age, and macrosomia compared to nondiabetic controls. These results are consistent with previous studies that have shown that women with diabetes are more likely to have poor perinatal and neonatal outcomes. In addition, these results are surprising because the study population did not have a high level of glycated hemoglobin (HbAlc) (6.9\% in the first trimester). Importantly, other maternal and neonatal outcomes were worse in pregnant women with type 2 diabetes than in those without diabetes, despite relatively low HbAic levels, although there were no differences in the risk for congenital malformations between the two groups.

Should we treat mild hyperglycemia in pregnant women with diabetes? In patients with type 1 diabetes, the risks for serious adverse outcomes and perinatal mortality were significantly higher when periconceptional HbA1c exceeded 6.9\% [10]. Furthermore, one retrospective study has shown that perinatal mortality in pregnant women with type 2 diabetes is four- and ninefold higher than in pregnant women with type 1 diabetes and in the background population, respectively [11]. 
Mean HbA1c levels were $6.8 \%$ in women with type 2 diabetes and $7.0 \%$ in those with type 1 diabetes, and glycemic control was similar or better in women with type 2 diabetes compared to women with type 1 diabetes. In addition, in the case of GDM, the Cochrane review states that there are various benefits of treating patients with mild GDM [12]. Consequently, we should recognize and manage mild hyperglycemia to reduce poor pregnancy outcomes in women with diabetes.

Fasting and postprandial monitoring of blood glucose are recommended to achieve metabolic and glycemic control in pregnant women with diabetes $[2,13]$. The American Diabetes Association and the Korean Diabetes Association recommend the following target blood glucose levels for women with diabetes: fasting, $<95$ $\mathrm{mg} / \mathrm{dL}$ (5.3 mmol/L); 1-hour postprandial, < $140 \mathrm{mg} / \mathrm{dL}$ (7.8 mmol/L); and 2-hour postprandial, < $120 \mathrm{mg} / \mathrm{dL}$ (6.7 $\mathrm{mmol} / \mathrm{L}$ ). The HbAlc target in pregnancy is $6 \%$ to $6.5 \%$ (42 to $48 \mathrm{mmol} / \mathrm{mol}$ ) [2,13].

All women of childbearing age with diabetes should be counseled on the importance of tight glycemic control prior to conception as well as during pregnancy. Because diabetic retinopathy may develop or progress during pregnancy, dilated eye examinations should be performed before pregnancy or in the first trimester, and then patients with pregestational diabetes should be monitored every trimester. Potentially, teratogenic medications such as angiotensin converting enzyme inhibitors, angiotensin receptor blockers, and statins should be avoided in women of childbearing age.

The prevalence of diabetes in pregnant women is increasing worldwide, and proper management is becoming increasingly important. Preconception counseling should be provided, and tight glycemic control should be achieved in women with diabetes. Even cases of mild hyperglycemia, despite the lack of symptoms, are associated with poor pregnancy outcomes in women with diabetes. Therefore, we should carefully treat mild hyperglycemia in pregnant women with diabetes.

\section{Conflict of interest}

No potential conflict of interest relevant to this article was reported.

\section{REFERENCES}

1. Chung SM, Moon JS. Simple screening using ultrasonography for prediction of gestational diabetes mellitus. Diabetes Metab J 2017;41:438-439.

2. American Diabetes Association. 13. Management of diabetes in pregnancy: standards of medical care in diabetes: 2018. Diabetes Care 2018;41(Suppl 1):S137-S143.

3. Jeon EJ, Hong SY, Lee J. Adipokines and insulin resistance according to characteristics of pregnant women with gestational diabetes mellitus. Diabetes Metab J 2017;41:457465.

4. Jovanovic L, Pettitt DJ. Gestational diabetes mellitus. JAMA 2001;286:2516-2518.

5. HAPO Study Cooperative Research Group, Metzger BE, Lowe LP, et al. Hyperglycemia and adverse pregnancy outcomes. N Engl J Med 2008;358:1991-2002.

6. Yang SH, Kim C, An HS, An H, Lee JS. Prediction of gestational diabetes mellitus in pregnant Korean women based on abdominal subcutaneous fat thickness as measured by ultrasonography. Diabetes Metab J 2017;41:486-491.

7. Guerin A, Nisenbaum R, Ray JG. Use of maternal GHb concentration to estimate the risk of congenital anomalies in the offspring of women with prepregnancy diabetes. Diabetes Care 2007;30:1920-1925.

8. Brydon P, Smith T, Proffitt M, Gee H, Holder R, Dunne F. Pregnancy outcome in women with type 2 diabetes mellitus needs to be addressed. Int J Clin Pract 2000;54:418-419.

9. Jang HJ, Kim HS, Kim SH. Maternal and neonatal outcomes in Korean women with type 2 diabetes. Korean J Intern Med 2018;33:1143-1149.

10. Jensen DM, Korsholm L, Ovesen P, et al. Peri-conceptional $\mathrm{AlC}$ and risk of serious adverse pregnancy outcome in 933 women with type 1 diabetes. Diabetes Care 2009;32:1046-1048.

11. Clausen TD, Mathiesen E, Ekbom P, Hellmuth E, Mandrup-Poulsen T, Damm P. Poor pregnancy outcome in women with type 2 diabetes. Diabetes Care 2005;28:323-328.

12. Alwan N, Tuffnell DJ, West J. Treatments for gestational diabetes. Cochrane Database Syst Rev 2009;(3):CDoo3395.

13. Korean Diabetes Association. 2015 Treatment Guidelines for Diabetes. $5^{\text {th }}$ ed. Seoul (KR): Gold' Planning and Development, 2015:35. 